\title{
Clinical utility gene card for: Non-Syndromic Microphthalmia Including Next-Generation Sequencing-Based Approaches
}

\author{
Rose Richardson $^{1}$, Jane Sowden ${ }^{2}$, Christina Gerth-Kahlert ${ }^{3}$, Anthony T Moore ${ }^{1,4,5}$ and Mariya Moosajee M $^{\star 1,5,6}$
}

European Journal of Human Genetics (2017) 25, doi:10.1038/ejhg.2016.201; published online 18 January 2017

\section{DISEASE CHARACTERISTICS}

1.1 Name of the disease (synonyms)

See Table 1, column 1-'Name of the disease'.

\subsection{OMIM\# of the disease}

See Table 1, column 2-'OMIM\# of the disease'.

1.3 Name of the analysed genes or DNA/chromosome segments and OMIM\# of the gene(s)

1.3.1 Core genes (irrespective if being tested by Sanger sequencing or next-generation sequencing)

See Table 1, column 4-'Associated gene(s)' and column 5-'OMIM\# of associated gene(s)'.

1.3.2 Additional genes (if tested by next-generation sequencing, including whole-exome/-genome sequencing and panel sequencing) See Table 2, column 1-'Gene' and column 3-'OMIM\# of gene'.

\subsection{Mutational spectrum}

Isolated microphthalmia is rare; most patients have associated ocular anomalies (complex), such as ocular coloboma, cataract and anterior segment dysgenesis. Nearly $80 \%$ of cases are associated with multisystemic features forming part of a syndrome. ${ }^{1-4}$ Only isolated and complex (non-syndromic) microphthalmia will be discussed (see Clinical Utility Gene Card for syndromic microphthalmia). There is a complex aetiology with chromosomal, monogenic and environmental causes identified. It is clinically and genetically heterogeneous, and may be inherited in an autosomal-dominant, -recessive or $\mathrm{X}$-linked recessive manner, although most cases of non-syndromic microphthalmia are sporadic. The occurrence of de novo mutations, mosaicism and incomplete penetrance makes prediction of the inheritance pattern difficult. Chromosomal duplications, deletions and translocations have been identified; a locus for autosomaldominant microphthalmia has been mapped to $15 \mathrm{q} 12-15,{ }^{5}$ and for autosomal-recessive microphthalmia at $14 \mathrm{q} 32 .{ }^{6,7}$ Autosomal-recessive VSX2 variants (causing MCOP2) account for $\sim 2 \%$ of isolated microphthalmia cases, and are predominantly missense. However, deletion of exon 3 has also been described..$^{8-10}$ Autosomal-recessive variants in RAX (MCOP3) ${ }^{10-12}$ and ALDH1A3 (MCOP8) can be missense, nonsense or frameshift, with some splice donor variants. A RAX gene deletion has also been described in one patient with bilateral anophthalmia, with no other ocular or systemic abnormalities reported. ${ }^{10} R A X$ mutations account for $\sim 2 \%$ of inherited anophthalmia/microphthalmia cases. ${ }^{11}$ Only missense variants have been found in GDF6 (MCOP4) ${ }^{10,13}$ and GDF3 (MCOP7), ${ }^{14}$ and are inherited in an autosomal-dominant manner. Homozygous or compound heterozygous variants in MFRP (MCOP5) ${ }^{15-19}$ or PRSS56 (MCOP6) ${ }^{20-23}$ are associated with autosomal-recessive posterior microphthalmia, which defines a rare distinct phenotype restricted primarily to the posterior segment of the eye. Patients with MFRP variants also develop a progressive rod cone dystrophy. ${ }^{18}$ Missense, nonsense and frameshift variants, plus splice donor variants have been described for both these genes.

COL4A1 (NM_001845.5: c.2317G > A (p.Gly773Arg); c.2122G > A (p.Gly708Arg)) variants are often reported in cases of brain small vessel disease with or without ocular anomalies, however, variants have been found in siblings with microphthalmia, other ocular anomalies, but no/few neurologic symptoms. ${ }^{24}$ Isolated microphthalmia with coloboma is a heterogeneous condition. Cases without systemic involvement are predominantly associated with autosomaldominant transmission, although a few recessive cases have been described. MCOPCB1 has been mapped to the X chromosome, ${ }^{25}$ and MCOPCB2 to chromosome 15q12-q15. ${ }^{26}$ Homozygosity for missense and a splice variant in VSX2 have been described in MCOPCB3 cases. $^{27,28}$ MCOPCB4 is isolated microphthalmia associated with colobomatous cyst and is transmitted as an autosomal-recessive trait. The genetic cause remains unknown. A heterozygous $24 \mathrm{bp}$ deletion in the coding region of $\mathrm{SHH}$ has been identified in MCOPCB5 patients. ${ }^{29,30}$ Heterozygous missense variants in $G D F 3^{14}$ and $A B C B 6^{31}$ have been described in patients with MCO PCB6 and MCOPCB7, respectively. Homozygous splice variant (NM_001080477.3: c.2968-2A > T (p.Val990Cysfs) $)^{32}$ and frameshift loss of function variant (NM_001080477.3: c.2083dupA $(\text { p.Thr695Asnfs) })^{33}$ in TENM3 result in MCOPCB9. A missense

${ }^{1}$ Department of Ocular Biology and Therapeutics, UCL Institute of Ophthalmology, London, UK; ${ }^{2}$ Department of Developmental Biology \& Genetics, UCL Institute of Child Health, London, UK; ${ }^{3}$ Department of Ophthalmology, University of Zurich, Zurich, Switzerland; ${ }^{4}$ Department of Ophthalmology, University of California, San Francisco, CA, USA; ${ }^{5}$ Department of Genetics, Moorfields Eye Hospital NHS Foundation Trust, London, UK; ${ }^{6}$ Department of Paediatric Ophthalmology, Great Ormond Street Hospital for Children NHS Foundation Trust, London, UK

*Correspondence: Dr M Moosajee, Department of Ocular Biology and Therapeutics, UCL Institute of Ophthalmology, 11-43 Bath Street, London EC1V 9EL, UK. Tel: +44 207608 6971; Fax: +44 207608 6830; E-mail: m.moosajee@ucl.ac.uk

Received 9 September 2016; revised 28 November 2016; accepted 14 December 2016; published online 18 January 2017 
Table 1 Overview of disease associated with non-syndromic (isolated and complex) microphthalmia

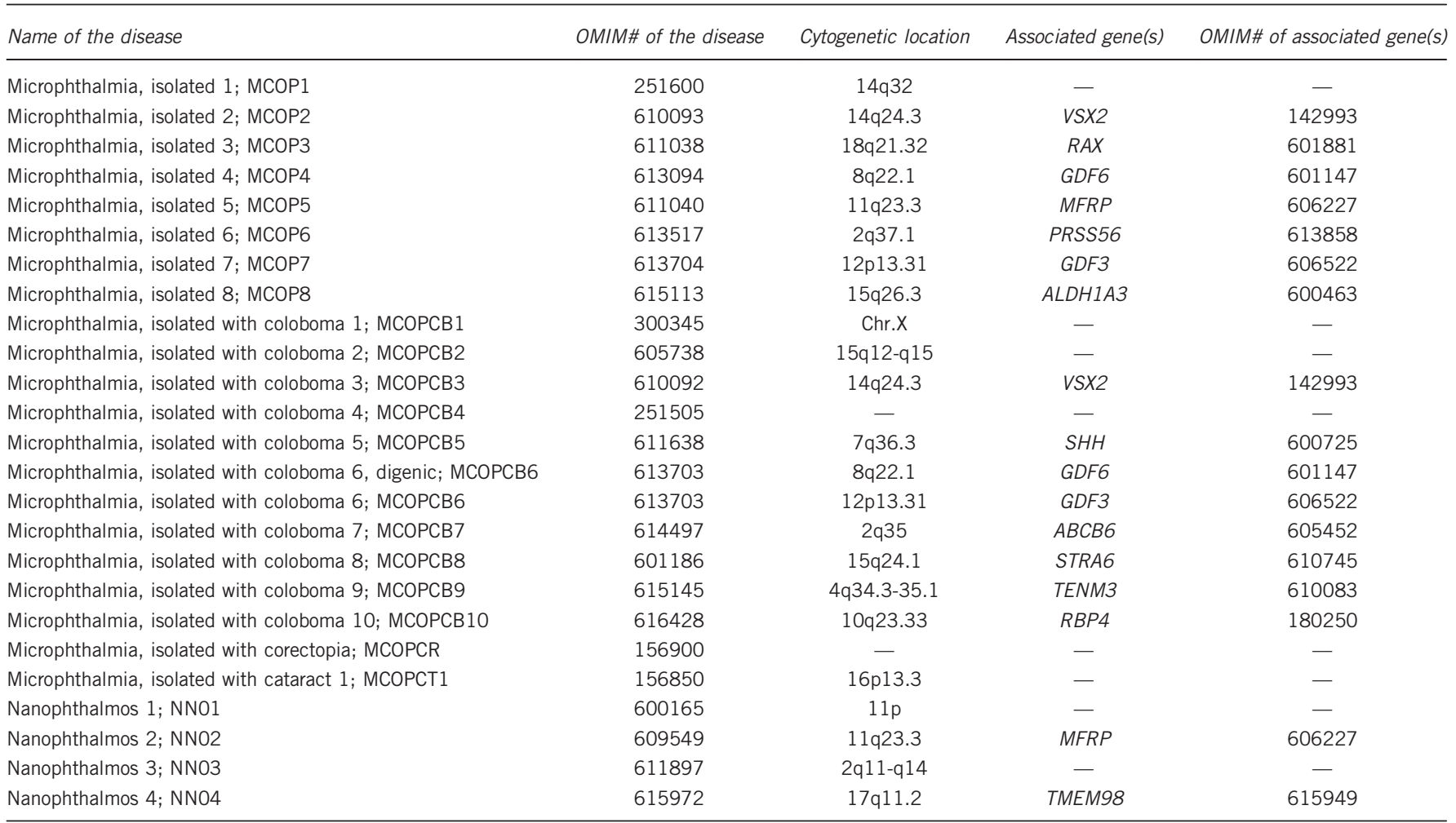

Table 2 Additional genes associated with isolated and complex microphthalmia, often with syndromic features, tested by next-generation sequencing

\begin{tabular}{|c|c|c|c|c|}
\hline Gene & Cytogenetic location & OMIM\# of gene & Associated disease acronym & OMIM\# of the disease (where applicable) \\
\hline BCOR & Xp11.4 & 300485 & Microphthalmia, syndromic 2 & 300166 \\
\hline BMP4 & $14 q 22.2$ & 112262 & Microphthalmia, syndromic 6 & 607932 \\
\hline CHD7 & $8 q 12.2$ & 605806 & CHARGE syndrome & 214800 \\
\hline COL4A1 & $13 q 34$ & 120130 & Brain small vessel disease with or without ocular anomalies & 607595 \\
\hline FREM 1 & $9 p 22.3$ & 608944 & Manitoba oculotrichoanal syndrome & 248450 \\
\hline HCCS & Xp22.2 & 300056 & Linear skin defects with multiple congenital anomalies 1 & 309801 \\
\hline HMGB3 & Xq28 & 300193 & Microphthalmia, syndromic 13 & 300915 \\
\hline$M A B 21 L 2$ & $4 q 31.3$ & 604357 & Microphthalmia, syndromic 14 & 615877 \\
\hline NAA10 & Xq28 & 300013 & Microphthalmia, syndromic 1 & 309800 \\
\hline OTX2 & $14 q 22.3$ & 600037 & Microphthalmia, syndromic 5 & 610125 \\
\hline PAX6 & $11 \mathrm{p} 13$ & 607108 & Ocular malformations within the $M A C$ spectrum & - \\
\hline$P X D N$ & $2 \mathrm{p} 25.3$ & 605158 & Cornea opacification and other ocular anomalies & 269400 \\
\hline$R A R B$ & $3 p 24.2$ & 180220 & Microphthalmia, syndromic 12 & 615524 \\
\hline SMOC1 & $14 q 24.2$ & 608488 & Microphthalmia with limb anomalies & 206920 \\
\hline sox2 & $3 q 26.33$ & 184429 & Microphthalmia, syndromic 3 & 206900 \\
\hline TMX3 & $18 q 22.1$ & 616102 & Microphthalmia with coloboma & - \\
\hline VAX1 & $10 q 25.3$ & 604295 & Microphthalmia, syndromic 11 & 614402 \\
\hline YAP1 & $11 q 22.1$ & 606608 & Ocular coloboma & 120433 \\
\hline
\end{tabular}

variant in RBP4 (NM_006744.3: c.217G > A (p.Ala73Thr)) has been associated with MCOPCB10. ${ }^{34}$

Many specific variants may cause varied phenotypes, for example, NM_001142617.1: c.1157G >A and c.1156G > A (p.Gly304Lys) in STRA6 causes MCOPCB8 (isolated microphthalmia and coloboma) and Matthew-Wood syndrome (bilateral anophthalmia with pulmonary agenesis and other associated systemic defects). ${ }^{35}$ Phenotypic findings in patients presenting with microphthalmia and congenital cataract (MCOPCT1) also include mental retardation and an individual with congenital heart disease. ${ }^{36,37}$ Patients with OTX2 variants have been described with specific hippocampal abnormalities, and phenotypic findings in patients affected by $R A X$ variants include 
developmental delay with autistic features and hypoplastic optic nerve and chiasm. ${ }^{11}$ MCOP4 has been reported in cases as isolated, or associated with skeletal anomalies, coloboma or polydactyly. Autism and cardiac anomalies have been described as additional features in a MCOP8-affected Pakistani patient, although these phenotypes may be unrelated to $A L D H 1 A 3$ variants. ${ }^{38}$ Furthermore, one patient with a variant in the $A L D H 1 A 3$ gene has been described with posterior coloboma and detached retina (NM_000693.2: c.568A > G (p.Lys190Ter)), and another with optic nerve and chiasm hypoplasia (NM_000693.2: c.1165A > T (p.Lys389Ter)) associated with MCOP8. ${ }^{39}$ This makes the genetic classification system of isolated/ complex and syndromic microphthalmia challenging.

A patient with a $2.7 \mathrm{Mb}$ deletion at 18q22.1, incorporating the gene TMX3, presented with microphthalmia. Two additional sequence variants have been identified in unrelated patients; a male with unilateral microphthalmia and retinal coloboma (NM_019022.3: c.116G >A (p.Arg39Gln)); and a female with unilateral microphthalmia and severe micrognathia (NM_019022.3: c.322G $>$ A, (p.Asp108Asn)) ${ }^{40}$ Consequently, the contribution of TMX3 variants to MCOPCB1 has been suggested, but remains to be confirmed.

Nanophthalmos is a subtype of simple microphthalmos. Autosomalrecessive nanophthalmos 2 (NNO2) has been associated with homozygosity for a nonsense (NM_031433.3: c.523C > T, (p.Gln175Ter)) or frameshift (NM_031433.3: c.1143insC (p. Gly383Ter)) variant, and compound heterozygosity for a frameshift (NM_031433.3: c.498delC (p.Asn167Thrfs)) or a missense (NM_031433.3: c.545 T > C (p.Ile182Thr)) variant in MFRP. ${ }^{41}$ Additional complications can develop, including angle-closure glaucoma, cystic oedema and retinal detachment. More recently, two segregating missense variants (NM_015544.2: c.577G >C (p.Ala193Pro); c.587A >C (p.His196Pro)) and a 34 bp heterozygous deletion (NM_015544.2: c.236_263+6del34) in TMEM98 have been described in autosomal-dominant nanophthalmos (NNO4) pedigrees. ${ }^{42,43}$

Of the monogenic causes of anophthalmia/microphthalmia, SOX2 has been implicated as a major causative gene, in which variants account for $15-20 \%$ of autosomal-dominant cases. ${ }^{44}$ However, patients with SOX2 variants usually present with other systemic malformations; the contribution of SOX2 variants to isolated microphthalmia specifically remains unknown. The majority of SOX2 sequence variants are de novo; nonsense, missense, frameshift and whole-gene deletions have been reported. ${ }^{10,45,46}$ Like SOX2, the majority of OTX2 variants are inherited nonsense and frameshift variants leading to haploinsufficiency, with some reports of whole-gene deletions. ${ }^{10,47}$ Patients often present with additional brain abnormalities. In view that variants in the genes listed in Table 2 cause a wide range of ocular phenotypes with different expressivity, their molecular screening must be recommended.

All data were mined from primary literature or curated genomic and phenotype databases, including ClinVar, public archive of interpretations of clinically relevant variants (http://www.ncbi.nlm. nih.gov/clinvar/); GeneReviews (http://www.ncbi.nlm.nih.gov/books/ NBK1116/); The Human Gene Mutation Database, HGMD (http:// www.hgmd.org/) and Online Mendelian Inheritance in Man, OMIM (http://omim.org/). Novel data should be shared through these databases. They were last accessed on 21 November 2016.

\subsection{Analytical validation}

Sequencing of both DNA strands. Disease-causing variants should be confirmed using genomic DNA from a new extraction. Causative variants found with next-generation sequencing should be verified using Sanger sequencing or other specific molecular methods (eg, PCR digest); for further details, see the Eurogentest Guideline. It is important to look for segregation to determine whether the variant is de novo in isolated cases, providing a higher likelihood it is pathogenic. In clinical practice, array comparative genomic hybridisation $(\mathrm{aCGH})$ or multiplex ligation-dependent probe amplification assay may be performed initially to detect deletions or duplications. Some molecular service labs also offer fluorescence in situ hybridisation to identify rearrangements or copy-number variation.

\subsection{Estimated frequency of the disease (incidence at birth ('birth} prevalence') or population prevalence)

If known to be variable between ethnic groups, please report:

The birth prevalence of microphthalmos ranges from 2 to 17 per $100000 .{ }^{48-53}$ In a prospective UK incidence study over 18 months, 135 confirmed cases of microphthalmia, anophthalmia and ocular coloboma (MAC) were reported in children under 16 years of age; microphthalmia was present in $66(48.9 \%)$ children; isolated in 31 $(23 \%)$ and mixed in $35(25.9 \%) .{ }^{54}$ Microphthalmia was reported in $3.2-11.2 \%$ of blind children worldwide in $2006{ }^{4}$

Epidemiological data suggest risk factors for microphthalmia are maternal age over 40, multiple births, infants of low birthweight and low gestational age. ${ }^{4,52,55}$ There is no predilection with regard to race or gender. ${ }^{52,55}$ Isolated microphthalmia is most commonly unilateral. ${ }^{55}$

\subsection{Diagnostic setting}

\begin{tabular}{lll}
\hline & Yes & No \\
A. (Differential) diagnostics & $\bigotimes$ & $\square$ \\
B. Predictive testing & $\bigotimes$ & $\square$ \\
C. Risk assessment in relatives & $\bigotimes$ & $\square$ \\
D. Prenatal & $\bigotimes$ & $\square$
\end{tabular}

Comment: Because of the time constraints, such as pregnancy, panel diagnostic or whole-exome sequencing, or whole-genome sequencing (WES/WGS) filtering is preferred if there is a request for prenatal diagnosis (which is rare).

\section{TEST CHARACTERISTICS}

\begin{tabular}{|c|c|c|c|c|}
\hline & \multicolumn{2}{|c|}{ Genotype or disease } & \multirow{2}{*}{$\begin{array}{l}\text { A: True positives } \\
\text { B: False positives }\end{array}$} & \multirow{2}{*}{$\begin{array}{l}\text { C: False negative } \\
\text { D: True negative }\end{array}$} \\
\hline & Present & Absent & & \\
\hline \multicolumn{5}{|l|}{ Test } \\
\hline \multirow[t]{2}{*}{ Positive } & $A$ & $\mathrm{~B}$ & Sensitivity: & $A /(A+C)$ \\
\hline & & & Specificity: & $D /(D+B)$ \\
\hline \multirow[t]{2}{*}{ Negative } & C & $\mathrm{D}$ & Positive predictive value: & $A /(A+B)$ \\
\hline & & & Negative predictive value: & $\mathrm{D} /(\mathrm{C}+\mathrm{D})$ \\
\hline
\end{tabular}

2.1 Analytical sensitivity

(proportion of positive tests if the genotype is present in the analyte)

\subsubsection{If tested by conventional Sanger sequencing}

Less than $100 \%$. The proportion is likely $<100 \%$, because primers may be localised on sequences containing SNVs or rare variants, which results in a preferential amplification of one allele (allele dropout). A supplementary deletion/duplication diagnostic test should be 
performed for genes with a known proportion of large genomic deletions/duplications as outlined in the section 'Analytical validation'.

\subsubsection{If tested by next-generation sequencing}

Less than $100 \%$. The proportion is likely $<100 \%$, because there might be disease-causing variants in regions that could not be enriched and/ or sequenced by next-generation sequencing owing to suboptimal coverage of some regions of interest with this technology, but depending on next-generation sequencing strategy. If ampliconbased enrichment strategies are being used, primers may be localised on SNVs or rare variants, which results in preferential amplification of one allele. In patients with a highly suggestive phenotype in whom testing for specific gene alterations proves negative, a supplementary deletion/duplication diagnostic test should be performed for genes with a known proportion of large genomic deletions/duplications as outlined in the section 'Analytical validation'.

\subsection{Analytical specificity}

(proportion of negative tests if the genotype is not present)

\subsubsection{If tested by conventional Sanger sequencing}

Nearly $100 \%$. False positives may at the most arise owing to misinterpretation of rare polymorphic variants.

\subsubsection{If tested by next-generation sequencing}

Less than $100 \%$. The risk of false positives owing to misinterpretation of rare polymorphic variants may be higher compared with Sanger sequencing because of greater number of analysed genes.

\subsection{Clinical sensitivity}

(proportion of positive tests if the disease is present)

The clinical sensitivity can be dependent on variable factors such as age or family history. In such cases, a general statement should be given, even if a quantification can only be made case by case.

\subsubsection{If tested by conventional Sanger sequencing}

Of those patients that undergo genetic testing of known causative genes with Sanger sequencing, $<10 \%$ of patients with isolated microphthalmia receive a molecular diagnosis and these are predominantly bilateral severe cases.

Most studies group microphthalmia with MAC, and therefore the most common causative genes are SOX2, OTX2, PAX6 and GDF6 contributing up to $10,3,2.5$ and $8 \%$, respectively. ${ }^{56}$ These are often syndromic cases and so the actual contribution to isolated microphthalmia is likely to be much lower.

\subsubsection{If tested by next-generation sequencing}

See section 'If tested by conventional Sanger sequencing'. Mutation detection rates are higher when combined WES with array aCGH and high-resolution analysis of intragenic microdeletions and microduplications are performed. WGS may aid in the detection of pathogenic variants in the promotor region, introns and other non-coding regulatory elements, and provide better coverage than exome sequencing. Regulatory element disruption in microphthalmia remains largely uncharacterised.

\subsection{Clinical specificity}

(proportion of negative tests if the disease is not present)

The clinical specificity can be dependent on variable factors such as age or family history. In such cases, a general statement should be given, even if a quantification can only be made case by case.

\subsubsection{If tested by conventional Sanger sequencing}

Unknown, however, if microphthalmia is not present, it is unlikely that a positive test will be detected.

\subsubsection{If tested by next-generation sequencing}

See section 'If tested by conventional Sanger sequencing'.

\subsection{Positive clinical predictive value}

(life time risk to develop the disease if the test is positive)

This is a congenital anomaly of the eye, therefore patients will be born with this defect, therefore nearly $100 \%$, however variable expressivity has been noted.

\subsection{Negative clinical predictive value}

(probability of not developing the disease if the test is negative)

Assume an increased risk based on family history for a non-affected person. Allelic and locus heterogeneity may need to be considered.

Index case in that family had been tested:

Nearly $100 \%$. If the non-affected relative is not a carrier of an identified disease-causing mutation, they have no increased risk, except a small risk related to the prevalence in the general population.

Index case in that family had not been tested:

Unknown.

\section{CLINICAL UTILITY}

3.1 (Differential) diagnostics: The tested person is clinically affected (To be answered if in 1.7 'A' was marked)

\subsubsection{Can a diagnosis be made other than through a genetic test?}

\begin{tabular}{lll}
\hline No. & $\square$ (continue with 3.1.4) \\
Yes & $\bigotimes$ & \\
& Clinically & $\square$ \\
& Imaging & $\square$ \\
& Endoscopy & $\square$ \\
& Biochemistry & $\square$ \\
& Electrophysiology & $\square$ \\
& Other (please describe)
\end{tabular}

\subsubsection{Describe the burden of alternative diagnostic methods to the patient}

The definition of microphthalmia is heterogenous, however, an axial length $(\mathrm{AL})$ of $<21 \mathrm{~mm}$ in adults and $<19 \mathrm{~mm}$ in a 1-year-old is most widely accepted as it represents a reduction of $2 \mathrm{SD}$ or more below normal. Microphthalmia can be detected using ultrasound during the second trimester, or after birth in conjunction with clinical examination. Microphthalmia can be associated with microcornea, which is defined as a horizontal diameter $<9 \mathrm{~mm}$ in a newborn and $<10 \mathrm{~mm}$ in children 2 years and older. Posterior microphthalmia is a rare subset of microphthalmia in which the total $\mathrm{AL}$ of the eyeball is reduced although anterior segment dimensions including corneal diameter, anterior chamber depth and anteroposterior length of the lens are normal, also detected by ultrasound. Nanophthalmia, a second rare subset of microphthalmia, is classically distinguished from posterior microphthalmia based on the presence of decreased anterior chamber dimensions.

Although a diagnosis of microphthalmia can be made relatively easily and cost-effectively, if this anomaly is seen, children should be investigated within a multidisciplinary team, including paediatricians and clinical geneticists, to ensure this is not part of a syndrome. 
Further monitoring may be required as syndromic manifestations may present later in childhood.

\subsubsection{How is the cost effectiveness of alternative diagnostic methods to be judged?}

Clinical examination and ultrasound imaging provides a cost-effective diagnosis.

\subsubsection{Will disease management be influenced by the result of a genetic test?}

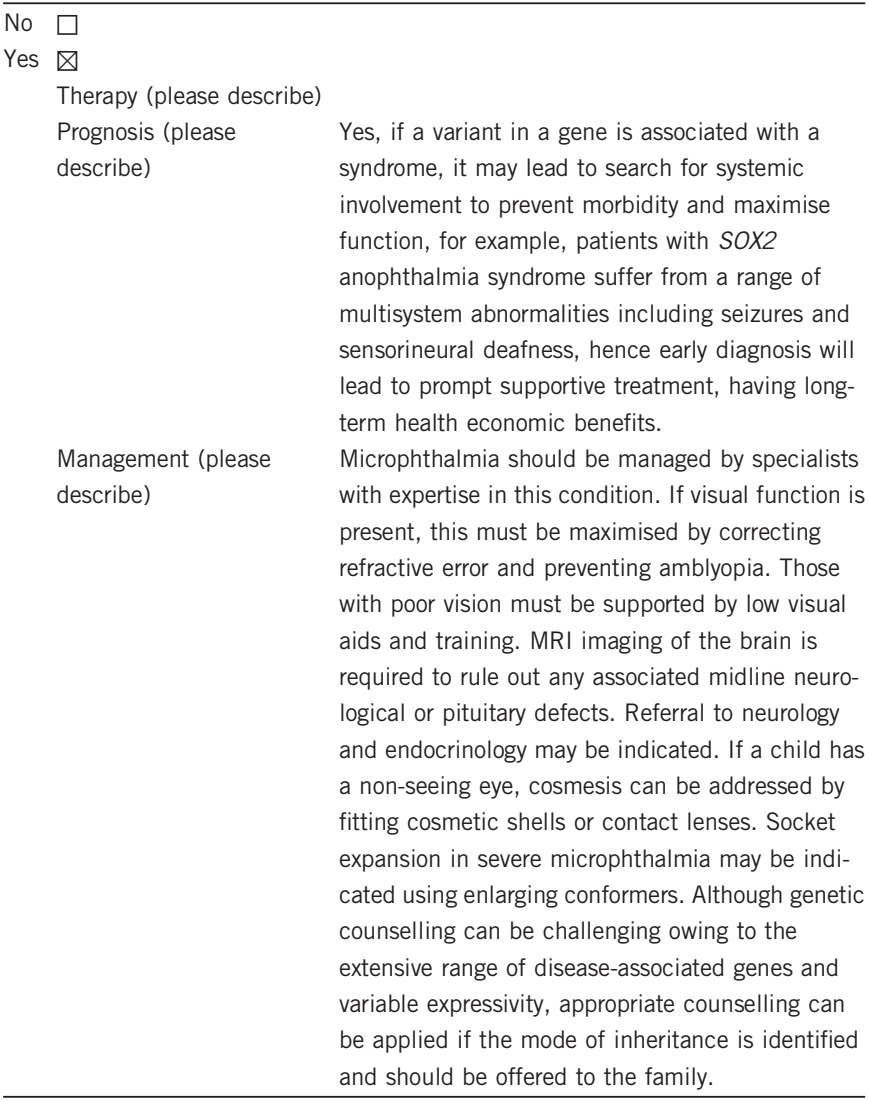

3.2 Predictive Setting: The tested person is clinically unaffected but carries an increased risk based on family history

(To be answered if in 1.7 'B' was marked)

\subsubsection{Will the result of a genetic test influence lifestyle and} prevention?

If the test result is positive (please describe)

Microphthalmia is a congenital eye anomaly, therefore if it is not clinically present at birth then this will not develop later in life. However, if an individual is clinically unaffected but is a carrier, this information will inform family planning if the mode of inheritance can be identified.

If the test result is negative (please describe) clinically unaffected person has a negative test result, no further follow-up is required. The result will inform family planning.
3.2.2 Which options in view of lifestyle and prevention does a person at-risk have if no genetic test has been done (please describe)? Vision can be variably affected in microphthalmic patients depending on the severity of the anomaly and the other complex features. This may limit schooling and professions that require perfect vision. Hence, a clinically confirmed diagnosis can help to provide guidance on career choice.

3.3 Genetic risk assessment in family members of a diseased person (To be answered if in 1.7 'C' was marked)

\subsubsection{Does the result of a genetic test resolve the genetic situation in} that family?

Yes, although there may be variable expressivity, non-penetrance and germline mosaicism, which will complicate the advice that can be given.

3.3.2 Can a genetic test in the index patient save genetic or other tests in family members?

If a disease-causing mutation is identified in the index patient, family members can be tested, but ophthalmic examination is also helpful. Test negative family members, who are clinically unaffected, do not need any further investigation or monitoring.

3.3.3 Does a positive genetic test result in the index patient enable a predictive test in a family member?

Yes, if the variant is known.

3.4 Prenatal diagnosis

(To be answered if in 1.7 'D' was marked)

3.4.1 Does a positive genetic test result in the index patient enable a prenatal diagnosis?

Yes. Germline mosaicism and/or variable penetrance render the prediction of recurrence risk difficult in monogenic microphthalmic individuals, however, molecular genetic studies for known variants are possible on amniotic fluid foetal cells withdrawn after 14 weeks of gestation or on chronic villus sampling at 10-12 weeks gestation, and can facilitate the diagnosis of microphthalmia. In addition, transvaginal ultrasonography enables the detection of microphthalmia from 12 weeks gestation $;{ }^{57}$ the maximal coronal or axial planes of the orbit are measured, and compared with established eye growth charts. ${ }^{58}$

\section{IF APPLICABLE, FURTHER CONSEQUENCES OF TESTING}

Please assume that the result of a genetic test has no immediate medical consequences. Is there any evidence that a genetic test is nevertheless useful for the patient or his/her relatives? (Please describe)

Beyond potentially defining recurrence risk information dependent on the cause and mode of inheritance, identifying the genetic aetiology may guide genetic counselling. It also contributes to the classification of syndromic or non-syndromic microphthalmia, thereby guiding any subsequent investigations for affected patients.

\section{CONFLICT OF INTEREST}

The authors declare no conflict of interest.

\section{ACKNOWLEDGEMENTS}

This work was supported by EuroGentest2 (Unit 2: 'Genetic testing as part of health care'), a Coordination Action under FP7 (grant agreement number 261469) and the European Society of Human Genetics. MM gratefully acknowledges the support of the National Institute for Health Research (NIHR) Biomedical Research Centre based at Moorfields Eye Hospital NHS Foundation Trust and UCL Institute of Ophthalmology. 
1 Verma AS, Fitzpatrick DR: Anophthalmia and microphthalmia. Orphanet J Rare Dis 2007; 2: 47.

2 Weiss AH, Kousseff BG, Ross EA, Longbottom J: Complex microphthalmos. Arch Ophthalmol 1989; 107: 1619-1624.

3 Nishina S, Kurosaka D, Nishida Y, Kondo H, Kobayashi Y, Azuma N: Survey of microphthalmia in Japan. Jpn J Ophthalmol 2012; 56: 198-202.

4 Forrester MB, Merz RD: Descriptive epidemiology of anophthalmia and microphthalmia, Hawaii, 1986-2001. Birth Defects Res A Clin Mol Teratol 2006; 76: 187-192.

5 Morle L, Bozon M, Zech JC et al: A locus for autosomal dominant colobomatous microphthalmia maps to chromosome 15q12-q15. Am J Hum Genet 2000; 67: 1592-1597.

6 Bessant DA, Khaliq S, Hameed A et al: A locus for autosomal recessive congenital microphthalmia maps to chromosome 14q32. Am J Hum Genet 1998; 62: 1113-1116.

7 Bessant DA, Anwar K, Khaliq S et al: Phenotype of autosomal recessive congenital microphthalmia mapping to chromosome 14q32. Br J Ophthalmol 1999; 83: 919-922.

8 Ferda Percin E, Ploder LA, Yu JJ et al: Human microphthalmia associated with mutations in the retinal homeobox gene CHX10. Nat Genet 2000; 25: 397-401.

9 Bar-Yosef U, Abuelaish I, Harel T, Hendler N, Ofir R, Birk OS: CHX10 mutations cause non-syndromic microphthalmia/anophthalmia in Arab and Jewish kindreds. Hum Genet 2004; 115: 302-309.

10 Chassaing N, Causse A, Vigouroux A et al: Molecular findings and clinical data in a cohort of 150 patients with anophthalmia/microphthalmia. Clin Genet 2014; 86: 326-334.

11 Voronina VA, Kozhemyakina EA, O'Kernick CM et al: Mutations in the human RAX homeobox gene in a patient with anophthalmia and sclerocornea. Hum Mol Genet 2004; 13: 315-322.

12 Lequeux L, Rio M, Vigouroux A et al: Confirmation of RAX gene involvement in human anophthalmia. Clin Genet 2008; 74: 392-395.

13 Asai-Coakwell M, French CR, Ye M et al: Incomplete penetrance and phenotypic variability characterize Gdf6-attributable oculo-skeletal phenotypes. Hum Mol Genet 2009; 18: 1110-1121.

14 Ye M, Berry-Wynne KM, Asai-Coakwell M et al: Mutation of the bone morphogenetic protein GDF3 causes ocular and skeletal anomalies. Hum Mol Genet 2010; 19: 287-298.

15 Ayala-Ramirez R, Graue-Wiechers F, Robredo V, Amato-Almanza M, Horta-Diez I, Zenteno JC: A new autosomal recessive syndrome consisting of posterior microphthalmos, retinitis pigmentosa, foveoschisis, and optic disc drusen is caused by a MFRP gene mutation. Mol Vis 2006; 12: 1483-1489.

16 Crespí J, Buil JA, Bassaganyas F et al: A novel mutation confirms MFRP as the gene causing the syndrome of nanophthalmos-renititis pigmentosa-foveoschisis-optic disk drusen. Am J Ophthalmol 2008; 146: 323-8.e1.

17 Zenteno JC, Buentello-Volante B, Quiroz-Gonzalez MA, Quiroz-Reyes MA: Compound heterozygosity for a novel and a recurrent MFRP gene mutation in a family with the nanophthalmos-retinitis pigmentosa complex. Mol Vis 2009; 15: 1794-1798.

18 Mukhopadhyay R, Sergouniotis PI, Mackay DS et al: A detailed phenotypic assessment of individuals affected by MFRP-related oculopathy. Mol Vis 2010; 16: 540-548.

19 Aldahmesh MA, Nowilaty SR, Alzahrani F et al: Posterior microphthalmos as a genetically heterogeneous condition that can be allelic to nanophthalmos. Arch Ophthalmol 2011; 129: 805-807.

20 Gal A, Rau I, El Matri L et al: Autosomal-recessive posterior microphthalmos is caused by mutations in PRSS56, a gene encoding a trypsin-like serine protease. Am J Hum Genet 2011; 88: 382-390.

21 Hmani-Aifa M, Ben Salem S, Benzina Z et al: A genome-wide linkage scan in Tunisian families identifies a novel locus for non-syndromic posterior microphthalmia to chromosome 2q37.1. Hum Genet 2009; 126: 575.

22 Nowilaty SR, Khan AO, Aldahmesh MA, Tabbara KF, Al-Amri A, Alkuraya FS: Biometric and molecular characterization of clinically diagnosed posterior microphthalmos. Am J Ophthalmol 2013; 155: 361-72.e7.

23 Orr A, Dube MP, Zenteno JC et al: Mutations in a novel serine protease PRSS56 in families with nanophthalmos. Mol Vis 2011; 17: 1850-1861.

24 Deml B, Reis LM, Maheshwari M, Griffis C, Bick D, Semina EV: Whole exome analysis identifies dominant COL4A1 mutations in patients with complex ocular phenotypes involving microphthalmia. Clin Genet 2014; 86: 475-481.

25 Lehman DM, Sponsel WE, Stratton RF et al: Genetic mapping of a novel X-linked recessive colobomatous microphthalmia. Am J Med Genet 2001; 101: 114-119.

26 Morlé L, Bozon M, Zech JC et al: A locus for autosomal dominant colobomatous microphthalmia maps to chromosome 15q12-q15. Am J Hum Genet 2000; 67: 1592-1597.

27 Ferda Percin E, Ploder LA, Yu JJ et al: Human microphthalmia associated with mutations in the retinal homeobox gene CHX10. Nat Genet 2000; 25: 397-401.

28 Bar-Yosef U, Abuelaish I, Harel T, Hendler N, Ofir R, Birk OS: CHX1O mutations cause non-syndromic microphthalmia/anophthalmia in Arab and Jewish kindreds. Hum Genet 2004: 115: 302-309.

29 Schimmenti LA, de la Cruz J, Lewis RA et al: Novel mutation in sonic hedgehog in nonsyndromic colobomatous microphthalmia. Am J Med Genet A 2003; 116a: 215-221.
30 Bakrania P, Ugur Iseri SA, Wyatt AW et al: Sonic hedgehog mutations are an uncommon cause of developmental eye anomalies. Am J Med Genet A 2010; 152a: 1310-1313.

31 Wang L, He F, Bu J et al: ABCB6 mutations cause ocular coloboma. Am J Hum Genet 2012; 90: 40-48.

32 Chassaing N, Ragge N, Plaisancié J et al: Confirmation of TENM3 involvement in autosomal recessive colobomatous microphthalmia. Am J Med Genet A 2016; 170: 1895-1898.

33 Aldahmesh MA, Mohammed JY, Al-Hazzaa S, Alkuraya FS: Homozygous null mutation in ODZ3 causes microphthalmia in humans. Genet Med 2012; 14: 900-904.

34 Chou CM, Nelson C, Tarle SA et al: Biochemical basis for dominant inheritance, variable penetrance, and maternal effects in RBP4 congenital eye disease. Cell 2015; 161: 634-646.

35 Casey J, Kawaguchi R, Morrissey $\mathrm{M}$ et al: First implication of STRA6 mutations in isolated anophthalmia, microphthalmia, and coloboma: a new dimension to the STRA6 phenotype. Hum Mutat 2011; 32: 1417-1426.

36 Zeiter HJ: Congenital microphthalmos. A pedigree of four affected siblings and an additional report of fortyfour sporadic cases. Am J Ophthalmol 1963; 55: 910-922.

37 Capella JA, Kaufman HE, Lill FJ: Hereditary cataracts and microphthalmia. Am J Ophthalmol 1963; 56: 454-458.

38 Fares-Taie L, Gerber S, Chassaing $\mathrm{N}$ et al: ALDH1A3 mutations cause recessive anophthalmia and microphthalmia. Am J Hum Genet 2013; 92: 265-270.

39 Yahyavi M, Abouzeid H, Gawdat $\mathrm{G}$ et al: ALDH1A3 loss of function causes bilateral anophthalmia/microphthalmia and hypoplasia of the optic nerve and optic chiasm. Hum Mol Genet 2013; 22: 3250-3258.

40 Chao R, Nevin L, Agarwal P et al: A male with unilateral microphthalmia reveals a role for TMX3 in eye development. PLoS One 2010; 5: e10565.

41 Sundin $\mathrm{OH}$, Leppert GS, Silva ED et al: Extreme hyperopia is the result of null mutations in MFRP, which encodes a Frizzled-related protein. Proc Natl Acad Sci USA 2005; 102: 9553-9558.

42 Awadalla MS, Burdon KP, Souzeau E et al: Mutation in TMEM98 in a large white kindred with autosomal dominant nanophthalmos linked to 17p12-q12. JAMA Ophthalmol 2014; 132: 970-977.

43 Khorram D, Choi M, Roos BR et al: Novel TMEM98 mutations in pedigrees with autosomal dominant nanophthalmos. Mol Vis 2015; 21: 1017-1023.

44 Williamson KA, FitzPatrick DR: The genetic architecture of microphthalmia, anophthalmia and coloboma. Eur J Med Genet 2014; 57: 369-380.

45 Williamson KA, FitzPatrick DR: SOX2-related eye disorders; in Pagon RA, Adam MP, Ardinger $\mathrm{HH}$ et al. (eds): GeneReviews. Seattle: University of Washington, 1993.

46 Bakrania P, Robinson DO, Bunyan DJ et al: SOX2 anophthalmia syndrome: 12 new cases demonstrating broader phenotype and high frequency of large gene deletions. $\mathrm{Br}$ J Ophthalmol 2007; 91: 1471-1476.

47 Wyatt A, Bakrania P, Bunyan DJ et al: Novel heterozygous OTX2 mutations and whole gene deletions in anophthalmia, microphthalmia and coloboma. Hum Mutat 2008; 29: E278-E283.

48 Bermejo E, Martinez-Frias ML: Congenital eye malformations: clinical-epidemiological analysis of 1,124,654 consecutive births in Spain. Am J Med Genet 1998; 75: 497-504.

49 Clementi M, Tenconi R, Bianchi F et al: Congenital eye malformations: a descriptive epidemiologic study in about one million newborns in Italy. Birth Defects Orig Artic Ser 1996; 30: 413-424.

50 Spagnolo A, Bianchi F, Calabro A et al: Anophthalmia and benomyl in Italy: a multicenter study based on 940,615 newborns. Reprod Toxicol 1994; 8: 397-403.

51 Kallen B, Tornqvist K: The epidemiology of anophthalmia and microphthalmia in Sweden. Eur J Epidemiol 2005; 20: 345-350.

52 Shaw GM, Carmichael SL, Yang W, Harris JA, Finnell RH, Lammer EJ: Epidemiologic characteristics of anophthalmia and bilateral microphthalmia among 2.5 million births in California, 1989-1997. Am J Med Genet A 2005; 137: 36-40.

$53 \mathrm{Hu}$ DN: Prevalence and mode of inheritance of major genetic eye diseases in China. J Med Genet 1987; 24: 584-588.

54 Shah SP, Taylor AE, Sowden JC et al: Anophthalmos, microphthalmos, and typical coloboma in the United Kingdom: a prospective study of incidence and risk. Invest Ophthalmol Vis Sci 2011; 52: 558-564.

55 Kallen B, Robert E, Harris J: The descriptive epidemiology of anophthalmia and microphthalmia. Int J Epidemiol 1996; 25: 1009-1016.

56 Gonzalez-Rodriguez J, Pelcastre EL, Tovilla-Canales JL et al: Mutational screening of CHX10, GDF6, OTX2, RAX and SOX2 genes in 50 unrelated microphthalmiaanophthalmia-coloboma (MAC) spectrum cases. Br J Ophthalmol 2010; 94: $1100-1104$.

57 Chen CP, Wang KG, Huang JK et al: Prenatal diagnosis of otocephaly with microphthalmia/anophthalmia using ultrasound and magnetic resonance imaging. Ultrasound Obstet Gynecol 2003; 22: 214-215.

58 Blazer S, Zimmer EZ, Mezer E, Bronshtein M: Early and late onset fetal microphthalmia. Am J Obstet Gynecol 2006; 194: 1354-1359. 